Supplement of Hydrol. Earth Syst. Sci., 23, 2261-2278, 2019

https://doi.org/10.5194/hess-23-2261-2019-supplement

(C) Author(s) 2019. This work is distributed under

the Creative Commons Attribution 4.0 License.

(c) (1)

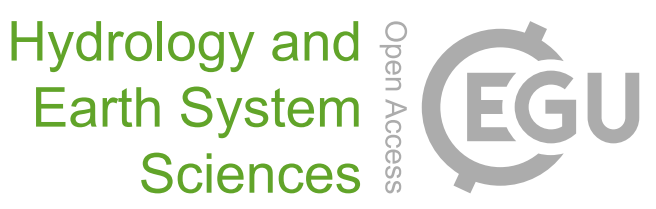

Supplement of

\title{
Using a coupled agent-based modeling approach to analyze the role of risk perception in water management decisions
}

\author{
Jin-Young Hyun et al. \\ Correspondence to: Yi-Chen Ethan Yang (yey217@lehigh.edu)
}

The copyright of individual parts of the supplement might differ from the CC BY 4.0 License. 


\section{Text S1 - Bayesian Inference Mapping Application in the Agent-Based Model}

In the field of water resource management, a decision is often made based on whether the preceding factor is larger (or less) than a prescribed threshold (i.e., exceedance). A simple example is that a farmer' belief of changing the irrigation area will be affected by the forecast of water stored in an upstream reservoir at the beginning of the growing season (i.e., water availability). In this study, both the forecast of a certain preceding factor $f$ (a random variable) and an agent's belief of taking a specific management behavior (or making a decision) $\theta$ can be represented as probabilities shown in Equations (S1) and (S2):

$$
\begin{aligned}
& P(f)=\frac{\# \text { of events that a preceding factor exceeds threshold }}{\# \text { of total events in modeling period }} \\
& P(\theta)=\frac{\# \text { of events of taking a management action }(=\text { make decision })}{\# \text { of total events in modeling period }}
\end{aligned}
$$

The conditional probability as represented in Equation (S3) describes the probability of a preceding factor exceeding its threshold given a specific decision was made.

$$
P(f \mid \theta)=\frac{P(f \cap \theta)}{P(\theta)}
$$

The conditional probability obtained in Equation (S3) is then used to calculate the joint probability of both the preceding factor exceeding its threshold and a particular decision being made (Equation S4).

$$
P(\theta \cap f)=P(f \mid \theta) \times P(\theta)
$$

Alternatively, the joint probability can be computed with Equation (S5).

$$
P(f \cap \theta)=P(\theta \mid f) \times P(f)
$$

Since the left-hand side of Equation (S4) and (S5) are mathematically equivalent, we can write their right-hand side as

$$
P(f \mid \theta) \times P(\theta)=P(\theta \mid f) \times P(f)
$$

Rearranging Equation (S6) provides a solution to $P(\theta \mid f)$ by Equation (S7)

$$
P(\theta \mid f)=\frac{P(f \mid \theta) \times P(\theta)}{P(f)}
$$

The marginal probability can be written as:

$$
P(f)=P(f \cap \theta)+P\left(f \cap \theta^{c}\right)
$$

where $\theta^{c}$ means that the management behavior was not made. $P(f \cap \theta)$ is the probability of the preceding factor exceeding its threshold when the decision was made, while 
$P\left(f \cap \theta^{c}\right)$ is the probability of the preceding factor exceeding its threshold when the decision was not made. Substituting Equation (S8) into Equation (S7):

$$
P(\theta \mid f)=\frac{P(f \mid \theta) \times P(\theta)}{P(f \cap \theta)+P\left(f \cap \theta^{c}\right)}
$$

Equation (S9) can be rewritten by expanding $P(f \cap \theta)$ and $P\left(f \cap \theta^{c}\right)$,

$$
P(\theta \mid f)=\frac{P(f \mid \theta) \times P(\theta)}{P(f \mid \theta) P(\theta)+P\left(f \mid \theta^{c}\right) P\left(\theta^{c}\right)}
$$

where $P\left(\theta^{c}\right)=1-P(\theta)$ is the probability of not taking the management behavior $\theta$. In our case, the information of $f$ is coming from RiverWare to ABM and $\theta$ is the result the ABM sends back to RiverWare.

Equation (S9) represents the probability of $\theta$ being made when the preceding factor exceeds the given threshold. Similarily, $\theta$ being made when the preceding factor does not exceed the threshold $\left(f^{c}\right)$ may be expressed as

$$
P\left(\theta \mid f^{c}\right)=\frac{P\left(f^{c} \mid \theta\right) \times P(\theta)}{P\left(f^{c} \mid \theta\right) P(\theta)+P\left(f^{c} \mid \theta^{c}\right) P\left(\theta^{c}\right)}
$$

The overall probability of taking a management behavior $P(\theta)$ relying on the preceding factor $f$ can be written using the law of total probability

$$
P(\theta)=P(\theta \mid f) \times P(f)+P\left(\theta \mid f^{c}\right) \times P\left(f^{c}\right)
$$

A solution of $P(\theta)$ can be obtained by substituting Equations (10) and (11) into (12)

$$
\begin{aligned}
& P(\theta)=\frac{P(f \mid \theta) \times P(\theta)}{P(f \mid \theta) P(\theta)+P\left(f \mid \theta^{c}\right) P\left(\theta^{c}\right)} \times P(f) \\
& +\frac{P\left(f^{c} \mid \theta\right) \times P(\theta)}{P\left(f^{c} \mid \theta\right) P(\theta)+P\left(f^{c} \mid \theta^{c}\right) P\left(\theta^{c}\right)} \times P\left(f^{c}\right)
\end{aligned}
$$

A general form of Equation (13) can be written as (Shafiee-Jood et al., 2017)

$$
P(\theta)=\sum_{i} P\left(\theta \mid F_{i}\right) \times P\left(F_{i}\right)=\sum_{i} \frac{P\left(F_{i} \mid \theta\right) P(\theta)}{\sum_{j} P\left(F_{i} \mid \Theta_{j}\right) P\left(\Theta_{j}\right)} \times P\left(F_{i}\right)
$$

where $i$ is the index for preceding factor and $j$ is the index for management behavior, $F_{i} \in$ $\left[f, f^{c}\right]$, and $\Theta_{j} \in\left[\theta, \theta^{c}\right]$. In this study, we re-name the variables in Equation (13) as follows

$$
\left\{\begin{array}{l}
\Gamma_{p r}=P(\theta) \\
\Gamma_{p d}=P(f) \\
\lambda=P(f \mid \theta)
\end{array}\right.
$$

where $\Gamma_{p r}$ represents the decision maker or agent's prior belief of $\theta, \Gamma_{p d}$ the probabilistic forecast of preceding factor $f, \lambda$ the rate of acceptance of new information which 
represents a decision maker's belief about the received information from $f$ (belief of the forecast/measurement accuracy representing the degree of ambiguity of $f$ ).

By applying the BI theory to Equation (13) with the expressions in Equation (15), the agent's prior belief of $\theta, \Gamma_{p r}^{t}$ at time $t$ can be expressed as

$$
\Gamma_{p r}^{t}=\frac{\lambda \Gamma_{p r}^{t-1}}{\lambda \Gamma_{p r}^{t-1}+(1-\lambda)\left(1-\Gamma_{p r}^{t-1}\right)} \Gamma_{p d}^{t}+\frac{(1-\lambda) \Gamma_{p r}^{t-1}}{(1-\lambda) \Gamma_{p r}^{t-1}+\lambda\left(1-\Gamma_{p r}^{t-1}\right)}\left(1-\Gamma_{p d}^{t}\right)
$$

In Equation (16), the agent's prior belief of $\theta$ at timestep $t, \Gamma_{p r}^{t}$, is updated based on the prior belief at previous timestep $t-1, \Gamma_{p r}^{t-1}$, and new incoming information or forecast at time $t, \Gamma_{p d}^{t} . \Gamma_{p r}^{t}$ lies in between $\Gamma_{p r}^{t-1}$ and $\Gamma_{p d}$. Two extreme cases are described here. When $\lambda=1$, Equation (16) reduces to $\Gamma_{p r}^{t}=\Gamma_{p d}^{t}$, which indicates that the agent's belief of taking management behavior is purely based on the new incoming information, which corresponds to a risk-seeking decision maker. In contrast, when $\lambda=0.5$, Equation (16) becomes $\Gamma_{p r}^{t}=\Gamma_{p r}^{t-1}$ suggesting that a decision is made based on an agent's previous experiences alone (i.e., the decision maker's most recent experience). This means that we have a risk-averse decision maker who totally ignores the new incoming information (or no information arrived) and strictly makes his/her decision based on his/her previous belief. In this study, the $\Gamma_{p r}^{t}$ in Equation (16) at each time step is updated by applying the Bayesian probability theory to $\Gamma_{p r}$ between two consecutive time steps to take the temporal causality between the two decisions into account. 


\section{Text S2 - ODD+D Protocol of Agent-Based Model}

\section{Overview}

\subsection{Purpose}

The coupled model provides a framework to simulate the action of the agents (farmers' decision on the annual irrigation plan) and interact with the environment which is simulated by a River-routing and reservoir management: RiverWare. The model is built for decision makers.

\subsection{Entities, State Variables, and Scales}

This model is composed of 16 irrigation districts (defined as farmer agents) to investigate the humans' decisions of the irrigation area over the river basin. The RiverWare operates on a daily time step, for a period of 85 years (October 1, 1928, to September 30,2013). The ABM operates on an annual time step to interact with the RiverWare. Space is characterized in the irrigation area size.

\section{List of Agents}

\begin{tabular}{|c|l|c|}
\hline Agent Name & Sub-Group & $\begin{array}{c}\text { Initial Size } \\
\text { (acre) }\end{array}$ \\
\hline JicarillaIrr & Group1 (Upstream of Navajo Reservoir) & 700 \\
\hline NMPineRiverAreaIrr & Group1 (Upstream of Navajo Reservoir) & 1420 \\
\hline TwinRocks & Group2 (Animas River - Tributary of San Juan River) & 251.3 \\
\hline NMAnimasIrr & Group2 (Animas River - Tributary of San Juan River) & 9341.1 \\
\hline FarmingtonGlade & Group2 (Animas River - Tributary of San Juan River) & 700 \\
\hline EchoDitch & Group2 (Animas River - Tributary of San Juan River) & 1210 \\
\hline FarmersMutual & Group2 (Animas River - Tributary of San Juan River) & 3050 \\
\hline Ralston & Group2 (Animas River - Tributary of San Juan River) & 407.6 \\
\hline ArchuletaDitch & Group3 (Downstream of Navajo Reservoir) & 40 \\
\hline CitizenDitch & Group3 (Downstream of Navajo Reservoir) & 3940 \\
\hline TurleyDitch & Group3 (Downstream of Navajo Reservoir) & 205 \\
\hline Hammond & Group3 (Downstream of Navajo Reservoir) & 40 \\
\hline FruitlandAndCambridge & Group3 (Downstream of Navajo Reservoir) & 540 \\
\hline JewettValley & Group3 (Downstream of Navajo Reservoir) & 920 \\
\hline Hogback & Group3 (Downstream of Navajo Reservoir) & 2140 \\
\hline CudeiCanal & Group3 (Downstream of Navajo Reservoir) & 170 \\
\hline Total Number of Agents in San Juan River Basin & $\mathbf{1 6}$ \\
\hline Total Irrigation Area (initial) in San Juan River Basin & $\mathbf{2 5 0 7 5}$ \\
\hline
\end{tabular}

The state variables in the model include six system parameters that set a foundation for the farmers' decision-making (e.g. precipitation, reservoir elevation and flow violation). The agents have beliefs parameters in Bayesian Inference to quantify their believe on information and socioeconomic parameters which acts as external threshold in the CostLoss model.

List of Parameters

\begin{tabular}{|c|l|c|}
\hline Parameter & Note & $\begin{array}{c}\text { \# of } \\
\text { Parameters }\end{array}$ \\
\hline Upstream Precip. Threshold & Apply to all agents: 16 agents in mm & 1 \\
\hline $\begin{array}{c}\text { Animas River Precip. } \\
\text { Threshold }\end{array}$ & Apply to agents in group 2: six agents in mm & 1 \\
\hline $\begin{array}{c}\text { Downstream Precip. } \\
\text { Threshold }\end{array}$ & Apply to agents in group 3: eight agents in mm & 1 \\
\hline
\end{tabular}




\begin{tabular}{|c|l|c|}
\hline Navajo Reservoir Elevation & Elevation in feet & 1 \\
\hline \multirow{2}{*}{ Frequency of flow violation } & $\begin{array}{l}\text { Number of days which the flowrate is below } 500 \text { cfs } \\
\text { at the outlet of San Juan River Basin }\end{array}$ & 1 \\
\hline \multirow{2}{*}{ NIIP Diversion threshold } & One single parameter will be used for all agents. & 1 \\
\hline \multirow{2}{*}{ Cost/Loss Parameter (z) } & $\mathrm{z}=\frac{\text { Cost (Total Cost due to expansion) }}{\text { Loss (Loss of Earning) }}$ & 16 \\
\hline Irr. Area Increment & Range from -5\% 5\% & 16 \\
\hline \multirow{3}{*}{$\begin{array}{c}\text { Farmers' Belief }\left(\lambda_{\mathrm{i}}\right) \\
{[0.5 \leq \lambda \leq 1]}\end{array}$} & $\lambda:$ Upstream Precip. -> Navajo Res. Elev. & 16 \\
\cline { 2 - 3 } & $\lambda:$ Upstream Precip. -> Decision Irr. Area & 6 \\
\cline { 2 - 3 } & $\lambda:$ Animas Precip. -> Decision Irr. Area & 16 \\
\cline { 2 - 3 } & $\lambda:$ Downstream Precip. -> Decision Irr. Area & 16 \\
\cline { 2 - 3 } & $\lambda:$ Flow Violation -> Decision Irr. Area & $\mathbf{1 0 2}$ \\
\cline { 2 - 3 } & $\lambda:$ updating Farmer's belief & \\
\hline Total Number of Parameters & & \\
\hline
\end{tabular}

\subsection{Process overview and schedule}

The ABM-Riverware interaction is triggered at the end of every water year. The state variables for the ABM are updated from the. After the ABM computation, newly updated irrigation areas and corresponded water diversions are imported to the RiverWare.

\section{Design concepts}

\subsection{Theoretical and Empirical Background}

This ABM adapt the Theory of Planned Behavior (TPB) to simulate human decisionmaking processes. Bayesian Inference (BI) mapping joined with a Cost-Loss (CL) model are applied to quantify the TPB. Incorporating BI mapping into an ABM allows an agent's psychological thinking process to be specified by a cognitive map between decisions and relevant preceding factors that could affect decision-making. A risk perception parameter is used in the BI mapping to represent an agent's belief on the preceding factors. Integration of the CL model addresses an agent's behavior caused by changing socioeconomic conditions. Example cognitive mapping of one agent group is given in the following. We test this ABM in a watershed in New Mexico and calibrate it using historical irrigated data from the US Bureau of Reclamation.

Group3.b_Downstream of San Juan River (Navajo Reservoir, dry region) with Shortage Sharing

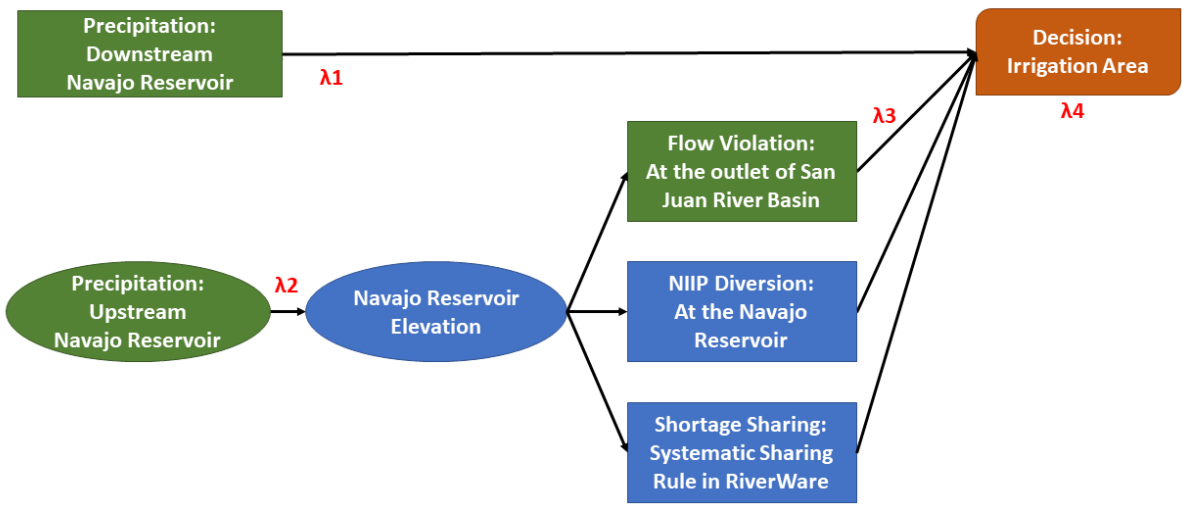




\subsection{Individual Decision-Making}

The objective of farmer agents is to make the decisions to expand or shrink their irrigated cropland on an annual time step, and these decisions derived from the causal probabilities (BI mapping) and following economic decision model, Cost-Loss (CL) model. In this model, no multiple level of decision-making included. The basic rationality behind agent decision-making in the model is that farmers will make decision based on their belief on incoming information as well as the socioeconomic condition at that time.

Farmers adapt their behavior to changing endogenous (their own belief " $\lambda$ " of different information) and exogenous (precipitation, reservoir elevation and flow violation) state variables in BI mapping based on the result of conditional probability calculation. Currently, we do not consider cultural value in the model. The spatial aspect is considered as multiple agents allocated in different places in the watershed and the temporal aspect is considered as the decision-making process is conduct for every year. The uncertainty is considered in the $\lambda$ as well as the randomness of irrigated areas increment of each agent.

\subsection{Learning}

The fundamental decision-making process of a farmer is based on the timely developing conditional probabilities (30 years of time window) in the BI mapping as well as the annual extremity. As time move on, this conditional probability will change since the 30 years of time window is different and this affect farmer agents' decision on irrigated areas.

\subsection{Individual Sensing}

The actual irrigated areas change action is made via the Cost-Loss Model and the CostLoss ratio (z) is an exogenous state variable (threshold) that agent will sense. This space scale of this Cost-Loss is considered for each agent group. In the current model, we assume agent know this variable and did not consider the costs for cognition and the costs for gathering this information.

\subsection{Individual Prediction}

The historical winter precipitation data from November to February in each year are used as a substitute for the snowpack forecast in this ABM model. This historical data assumed to be used as the perfect prediction of the snowpack which means we do not allow agents be erroneous in this prediction process.

\subsection{Interaction}

Agent interact indirectly through their decision on the water usage by changing their irrigation areas. Since agents' decisions change the managing plan in the RiverWare due to the dynamic change of the agricultural water usages, the interaction is depend on the RiverWare simulation. Current model does not have direct communication and systemwide coordination.

\subsection{Collectives}

In the case study, agents are grouped into three different regions (Collectives). The spatial variation of the precipitation (snowpack) is considered in different groups. Within a group, the agents share the same systematic parameters and BI structure. Collectives in the model are represented by different structure of agent's cognitive map. For instance, agents in 
Group 3: Downstream Navajo Reservoir, will be affected the reservoir's release schedule. But agents in Group 1: Upstream Navajo Reservoir, will make their decision without considering the reservoir operation.

\subsection{Heterogeneity}

Agents are defined heterogeneous in the model. As mentioned in 2.7, different groups of agents will have different cognitive maps. Also, the Cost-Loss ratios are different in different group to represent the heterogeneous socioeconomic condition. Each agent will consider precipitation differently as well. These heterogeneity are considered in each agent's decision-making process.

\subsection{Stochasticity}

Stochasticity is included in the model regarding the increasing or decreasing rate of the irrigation area. The percentile change with the $2 \%$ of maximum limitation is applied after the binary decision (0: decreasing and 1: increasing).

\subsection{Observation}

The simulated irrigation areas are validated by the historically observations of the irrigation area from the US Bureau of Reclamation. The output data from the ABM decisions, the areal increments and the actual water diversions, are used for updating the corresponding Object/Slow values in the RiverWare.

\section{Details}

\subsection{Implementation Details}

The ABM model is implemented in MATLAB and the RiverWare is a commercialized software which require license to execute. The MATLAB code will be available on the project's GitHub website. The corresponding author can also send the code to anyone who is interested in the model.

\subsection{Initialization}

The RiverWare, simulates the river-basin operation rules from October 1, 1928, to September 30, 2013 (water-year cycle). Before the simulation in the RiverWare, the model initialized all input flows (derived from hydrologic models - VIC and StateMob) and constant parameters (given by the river basin regulations and the reservoir operation) for internal calculations. The initialization is always the same during the simulation because the initialization performed once before the beginning of the RiverWare simulation. The ABM begins to interact with RiverWare at the end of water year (September 30 $\left.{ }^{\text {th }}, 1929\right)$. The initial sizes of the irrigation areas are taken from the historical observations from the US Bureau of Reclamation.

\subsection{Input Data}

Most of the input data-including stream flow at the outlet of the basin, the Navajo Reservoir elevation, the irrigation areas and the water diversion for Indian Reservation district (NIIP) for the ABM are retrieved from the RiverWare. On the other hand, the precipitation data are taken from external sources: ground-based rainfall observatories (rain-gauges) operated by National Oceanic Atmospheric Administration (NOAA). 
3.4. Sub-models

There is no sub-models in this study. 
Figure S1 to Figure S4

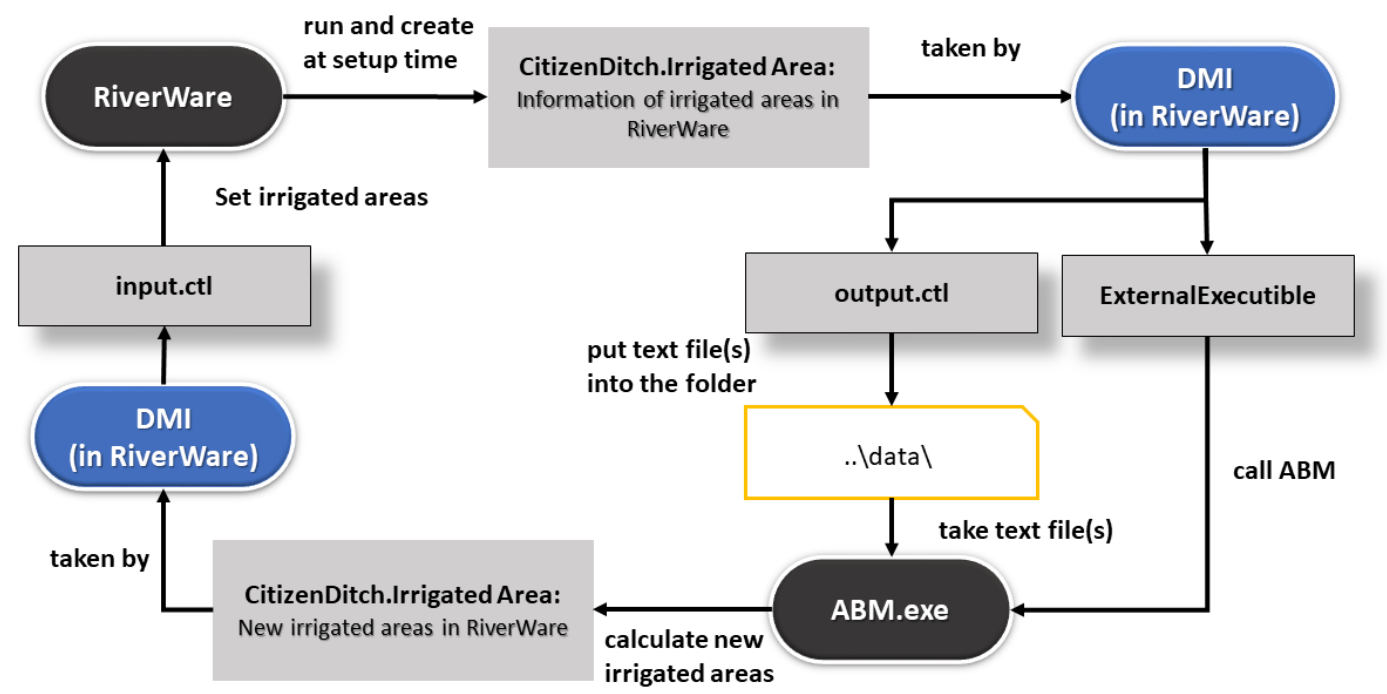

Figure S1. An illustration of the two-way coupling process between an agent-based model $(\mathrm{ABM})$ and a RiverWare using a built-in function of RiverWare: the data management interface (DMI). The figure uses a Water Use Object in RiverWare as an example. The DMI retrieves data from targeted Slots (e.g., irrigation area and water demand in CitizanDitch irrigation area) in RiverWare and exports the data (text files) to the ABM with the path assigned by the "output.ctl" control file. By using exported data and other inputs, the ABM makes the necessary calculations for simulating the human decisionmaking process (determine the new irrigation are and water demand for the coming year). The updated irrigation area and water demand are then input back to the same RiverWare Slots designated in the "input.ctl" control file. This process is repeated at the end of each water year throughout the model period. 
(a) Extremities: Upstream San Juan (Group1)

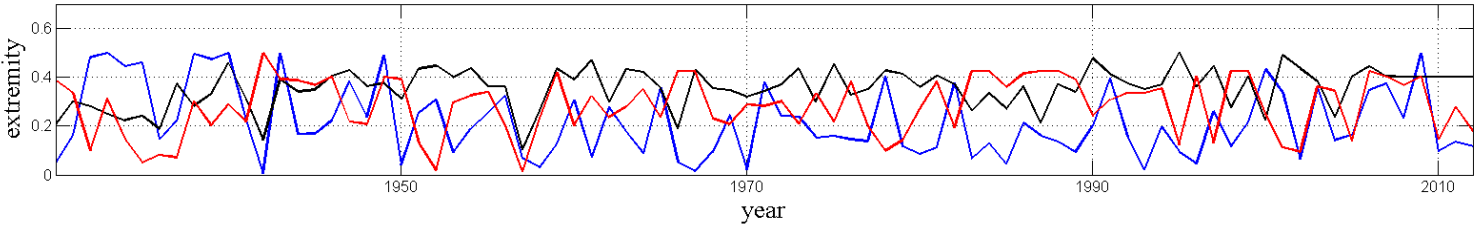

(b) Extremities: Animas river (Group2)

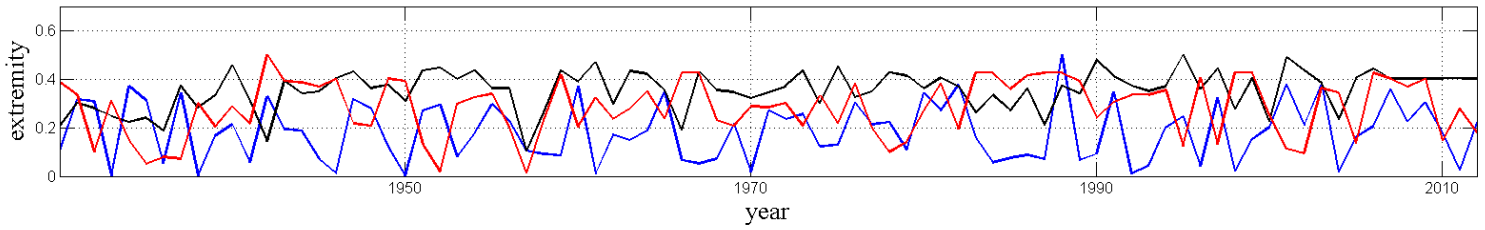

(c) Extremities: Downstream San Juan (Group3)

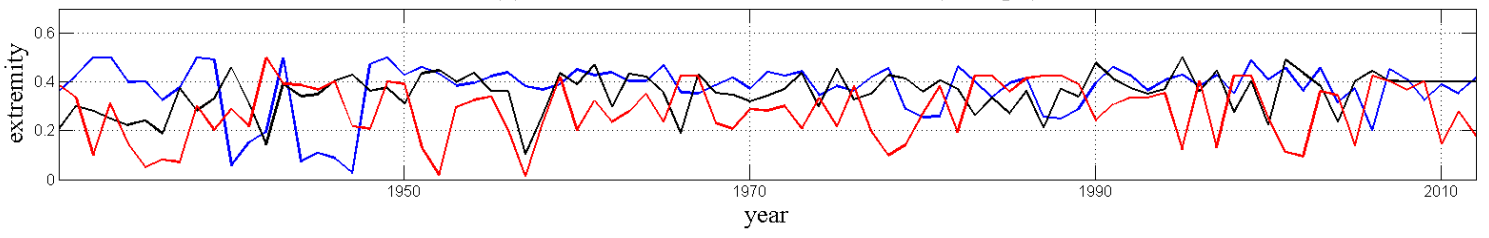

Figure S2. Extremities of preceding factor considered by (a) Upstream San Juan River, (b) Animas River, and (c) Downstream San Juan agents. 

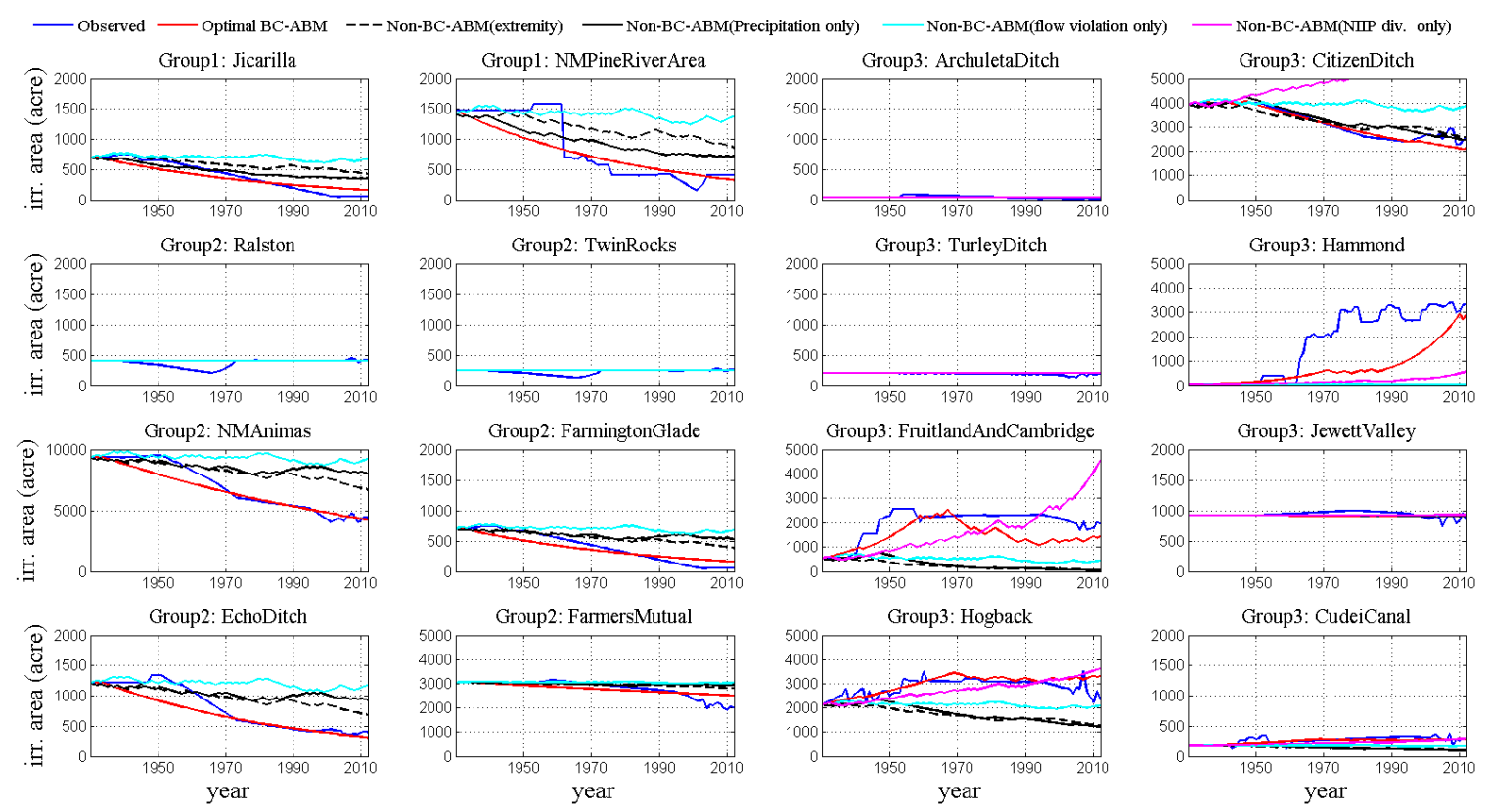

Figure S3. The simulated irrigation area changes during 1928 to 2013 from BC-ABM (solid red), Non-BC-ABM with extremity (dashed black), and Non-BC-ABM based on single preceding factor such as precipitation (solid black), flow violation (solid cyan), and NIIP diversion (solid magenta) versus historical irrigation areas (solid blue). 


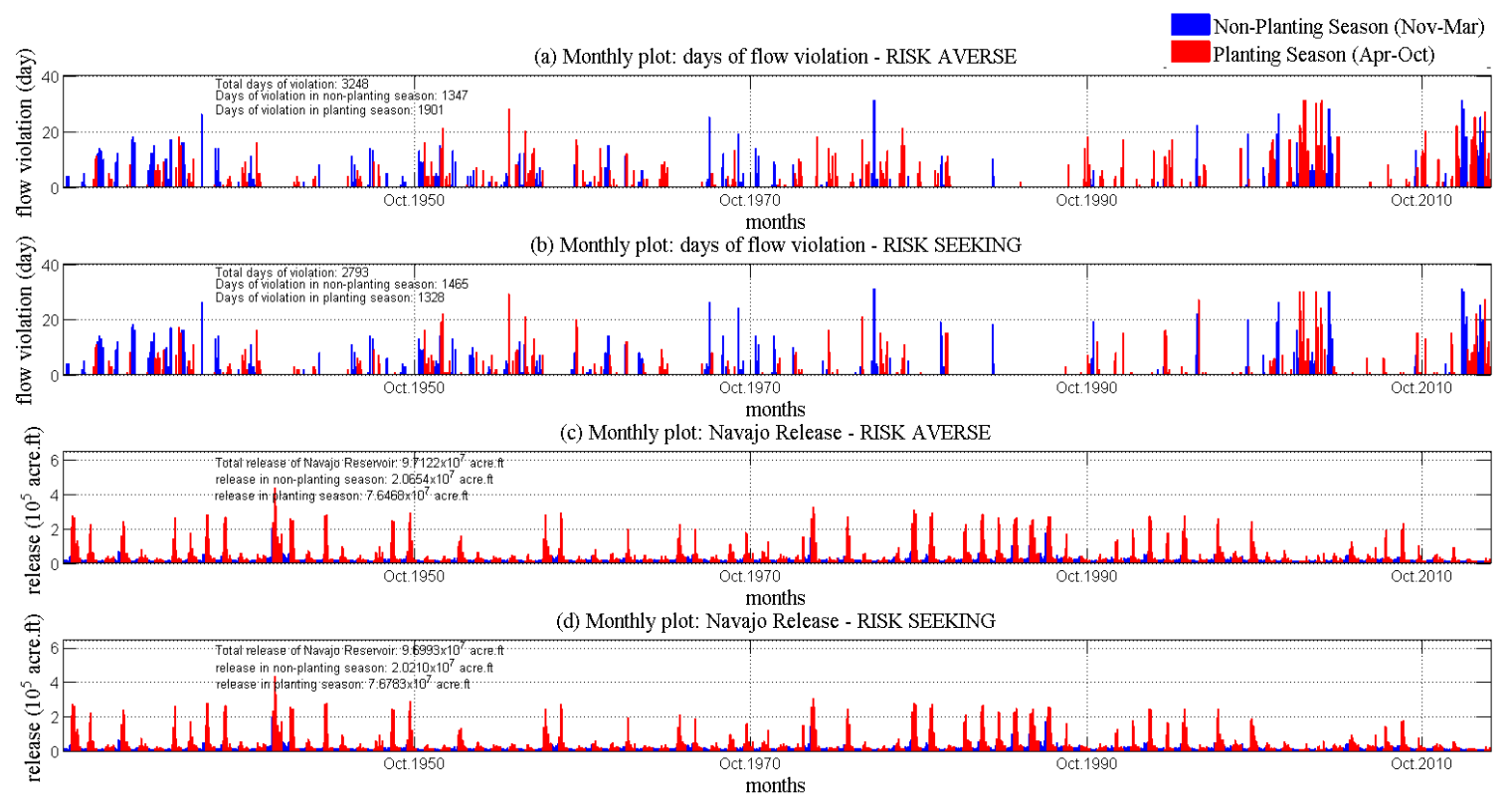

Figure S4. (a) Monthly view of days of flow violation with the case of the "Risk Averse"; (b) Monthly view of days of flow violation with the case of the "Risk Seeking"; (c) Monthly view of Navajo Reservoir Release with the case the "Risk Averse"; (d) Monthly view of Navajo Reservoir Release with the case of the "Risk Seeking" 


\section{References}

Grimm, V., Berger, U., DeAngelis, D. L., Polhill, J. G., Giske, J., and Railsback, S. F.: The ODD protocol: A review and first update. Ecol. Model., 221, 2760-2768, doi:10.1016/j.ecolmodel.2010.08.019, 2010.

Müller, B., Bohn, F., Dreßler, G., Groeneveld, J., Klassert, C., Martin, R., Schlüter, M., Schulze, J., Weise, H., and Schwarz, N.: Describing human decisions in agentbased models - ODD + D, an extension of the ODD protocol. Environ. Model. Softw, 48, 37-48, doi:10.1016/j.envsoft.2013.06.003, 2013. 